\title{
A First Step to a Biomarker of Curative Surgery in Colorectal Cancer by Liquid Biopsy of Methylated Septin 9 Gene
}

\author{
M. Leon Arellano $\mathbb{D}^{1},{ }^{1}$ M. García-Arranz $\mathbb{D}^{2},{ }^{2}$ R. Ruiz, ${ }^{3}$ R. Olivera, ${ }^{2}$ S. Magallares, ${ }^{3}$ \\ S. Olmedillas-Lopez $\mathbb{D}^{2},{ }^{2}$ T. Valdes-Sanchez, ${ }^{3}$ H. Guadalajara $\mathbb{D}^{\mathbb{D}},{ }^{1}$ and D. García-Olmo ${ }^{1}{ }^{1}$ \\ ${ }^{1}$ Department of Surgery, Hospital Fundación Jimenez Diaz, Madrid, Spain \\ ${ }^{2}$ New Therapy Laboratory, Instituto de Investigación Sanitaria Fundación Jiménez Díaz, Madrid, Spain \\ ${ }^{3}$ Bemygene, Valencia, Spain
}

Correspondence should be addressed to M. Leon Arellano; miguel.leon@quironsalud.es

Received 10 November 2019; Accepted 16 January 2020; Published 5 June 2020

Academic Editor: Monica Cantile

Copyright (c) 2020 M. Leon Arellano et al. This is an open access article distributed under the Creative Commons Attribution License, which permits unrestricted use, distribution, and reproduction in any medium, provided the original work is properly cited.

\begin{abstract}
Objectives. To confirm that patients affected by colorectal cancer have the V2 region of Septin 9 (SEPT9) gene hypermethylated in the circulating free DNA from a peripheral blood sample before surgery and to determine if this hypermethylated DNA disappears from the patients after complete resection of the tumour. Methods. Plasma from 10 patients with colorectal cancer was collected preoperative and three months after surgery. The analysis of the methylation status of the promoter region of the SEPT9 gene was performed using a 7500 Fast Real-Time PCR System. Results. Hypermethylation of SEPT9 gene was detected in 8 out of 10 preoperative samples (one negative result was probed to be a Lynch syndrome) and in 4 out of 10 postoperative samples matching with the cases of recurrence or persistence of disease. This means that, in this sample, the preoperative sensitivity and specificity of the test were $88.9 \%$ and $100 \%$, respectively, and there is $100 \%$ correlation between the positive results of the SEPT9 test and a recurrence/persistence of the disease in patients after surgical resection. Conclusions. Our study shows that circulating hypermethylated SEPT9 is a specific colorectal cancer biomarker. This hypermethylated SEPT9 DNA disappears around three months after surgery and that circulating hypermethylated SEPT9 may be the first noninvasive marker for postsurgical diagnosis; this conclusion must be confirmed with a more significant number of patients.
\end{abstract}

\section{Introduction}

Colorectal cancer (CRC) is one of the most frequent tumours and almost the leading cause of deaths among cancer worldwide. Despite the development of new treatments, most patients are first-time diagnosed at the middle or late stage of CRC, leading to high mortality and a poor prognosis. The surgical approach is the best treatment for patients with CRC, but recurrence or persistence after resection is associated with a severe prognosis [1].

Approximately $25 \%$ to $40 \%$ of patients who undergo curative resection of colorectal cancer (CRC) develop tumour recurrence with eventual demise [2]. An optimal surveillance protocol for CRC includes CT scans, colonoscopies, and serum carcinoembryonic antigen (CEA) measurement, although this blood marker has a reduced sensitivity or spec- ificity. Normal CEA values may be found in almost $50 \%$ of cancers before surgical resection and often do not rise during recurrences [3]. The primary objective of a surveillance protocol of CRC patients is to improve survival rates [4].

Therefore, this leads to a search for an accurate, convenient, and noninvasive biomarker that could detect persistence or recurrence of CRC, including also patients with a complete response in rectal cancer after neoadjuvant therapy, evaluation of complete resection after peritoneal oncological surgery, or local excision for early stage rectal cancer. With this tool, we could offer better surveillance and a rational adjuvant prescription.

The SEPT9 gene methylation assay, a blood-based test explicitly used for CRC detection and screening, was developed and used clinically in the last decade $[5,6]$. The test analyses the methylation status of the gamma promoter 
region of transcript V2 of the SEPT9 gene. Because it is known that CRC develops from the accumulation of changes at the genetic and epigenetic levels in the epithelial cells of the colon, molecular markers of genetic and epigenetic alterations in tumour tissues and peripheral blood have been evaluated. The hypermethylated SEPT9 gene has emerged as an accurate biomarker to detect CRC in peripheral blood and tumoural tissue $[7,8]$. This test analyses the methylation status of the gamma promoter region of the SEPT9 gene V2 transcript, which is differentially methylated in CRC patients. Septins are an evolutionarily conserved group of GTPbinding proteins that form filamentous hetero-oligomers, but their molecular role in tumorigenesis remains largely unknown [9-12]. Septin genes are reported to be essential in cell division, especially in cytokinesis, but they are also involved in membrane transport and fusion and exocytosis; they can also act as scaffolds that recruit other proteins or provide rigidity to cell membranes [13-15].

SEPT9 promoter is hypermethylated in CRC tissues but also can be detected in blood plasma, due to the presence of tumour DNA released from necrotic and apoptotic CRC cells $[6,7,16]$. Numerous validation studies analyzing the performance of the SEPT9 assay have been conducted; in particular, the FDA-approved test Epi proColon 2.0, developed by Epigenomics AG, reached sensitivity and specificity values ranging from $68 \%$ to $96 \%$ and from $80 \%$ to $97 \%$, respectively [17]. For these reasons, it has emerged as an accurate and noninvasive method for primary CRC detection. The Septin 9 assay was initially developed for the early diagnosis of CRC in population screening, especially for those reluctant to undergo colonoscopy. Later on, several reports have also suggested its usefulness in monitoring patient evolution following a therapeutic intervention or even as predictive markers of the response to chemo- and radiotherapy $[18,19]$.

Although the test was designed for early detection and screening of CRC, due its sensitivity and the high specificity found, the hypothesis of using it as a diagnostic tool as a biomarker of detection of curative surgery and as a recurrence follow-up protocol seems reasonable. We decided to perform a proof of concept study measuring the SEPT9 methylation level before and after surgery on ten patients with the following objectives:

(1) To assure if indeed those patients affected by CRC, before surgery, have the SEPT9 promoter hypermethylated in the circulating free DNA from a peripheral blood sample

(2) To check if SEPT9 hypermethylated DNA disappears after a complete resection of a tumour

(3) To determine the relationship between the maintenance of hypermethylated DNA after a complete resection of the tumour and the possible recurrence or persistence of CRC

\section{Material and Methods}

2.1. Patients and Sample Collection. A prospective study of 10 patients with CRC at a single institution (Hospital Universi-
TABLE 1: Criteria for the validity of the system according to manufacturer instructions. $\mathrm{Ct}$ is threshold amplification cycle. $A C T B$ is $\beta$-actin gene.

\begin{tabular}{lcc}
\hline Gene & Results & Conclusion \\
\hline SEPT9 & $\mathrm{Ct}<41.1$ & Control positive valid \\
ACTB & $\mathrm{Ct} \leq 29.8$ & \\
SEPT9 & $\mathrm{Ct}$ undetermined & Control negative valid \\
ACTB & $\mathrm{Ct} \leq 7.2$ & \\
\hline
\end{tabular}

tario Fundación Jimenez Diaz) was conducted between March 2017 and December 2017. The study was approved by the Ethics Committee Clinical Research (PIC number 157_2016_FJD), and all the patients included signed the informed consent prior the extraction of the samples.

Patients included in the study must meet the following inclusion criteria: a confirmed diagnosis of colorectal cancer, surgical treatment of a tumour without the need for a posterior ostomy, no previous treatment of chemotherapy or radiotherapy, age between 50 and 75 years, and signed informed consent.

Blood samples were collected at two points: preoperative and three months after surgery. All patients had the established protocol of postoperative CRC surveillance. It was recorded in the clinical history all the values associated with the characteristics and evolution of patients during the clinical follow-up (tumour stage, adverse events, clinical progression, and pain).

Ten $\mathrm{mL}$ of blood samples were extracted from each patient into potassium ethylenediaminetetraacetic acid (EDTA) tube and processed immediately (less 1 hour) by double centrifugation at $1400 \times \mathrm{g}$ for $12 \mathrm{~min}$. The plasma obtained was transferred into a new tube and directly stored at $-80^{\circ} \mathrm{C}$ for subsequent testing.

2.2. Analysis of the Methylation Status of Circulating SEPT9 DNA in Plasma. The circulating free DNA (cfDNA) from $3.5 \mathrm{~mL}$ of plasma was captured, concentrated, and bisulfiteconverted using the Epi proColon 2.0 Plasma Quick Kit following the instructions of the manufacturer (Epigenomics AG, Berlin, Germany). The ammonium bisulfite (Epi proColon Bisulfite Solution) converts unmethylated cytosines into uracils. The bisulfite-converted DNA (bisDNA) was then amplified in a duplex quantitative PCR (qPCR) using the Epi proColon Sensitive PCR kit. The kit includes specific primers for the promoter region of SEPT9 and ACTB ( $\beta$-actin), the latter as an internal control for DNA concentration, which hybridise to regions lacking $\mathrm{CpG}$ dinucleotides. The PCR reaction is based on a heavy methyl amplification, in which a bisulfite-converted unmethylated sequence-specific blocker prevents the amplification of unmethylated DNA, combined with the use of a methylated SEPT9-specific fluorescent detection probe [9]. The samples were amplified in triplicate using a 7500 Fast Real-Time PCR System (Thermo Fisher Scientific). Epi proColon positive and negative external controls were used in all independent runs.

We recorded PCR data from the 7500 Fast Dx software for $A C T B$ and methylated SEPT9 for each of the triplicate 
TABLE 2: Results and validity of preoperative and postoperative SEPT9 blood test.

\begin{tabular}{|c|c|c|c|c|c|c|}
\hline \multirow{2}{*}{ Patient } & \multicolumn{3}{|c|}{ Preoperative } & \multicolumn{3}{|c|}{ Postoperative } \\
\hline & SEPT9 $(\mathrm{Ct})$ & $A C T B(\mathrm{Ct})$ & Results & SEPT9 $(\mathrm{Ct})$ & $A C T B(\mathrm{Ct})$ & Results \\
\hline 1 & $36.1 \pm 0.1$ & $29.5 \pm 0.1$ & Positive & Undetermined & $27.7 \pm 0.0$ & Negative \\
\hline 2 & $32.2 \pm 0.3$ & $28.4 \pm 0.2$ & Positive & $40.6 \pm 0.1$ & $27.1 \pm 0.1$ & Positive \\
\hline 3 & $40.3 \pm 1.8$ & $29.1 \pm 0.1$ & Positive & Undetermined & $28.3 \pm 0.1$ & Negative \\
\hline 4 & $28 \pm 0.1$ & $29.0 \pm 0.1$ & Positive & $33.6 \pm 0.5$ & $26.5 \pm 0.1$ & Positive \\
\hline 5 & $36.9 \pm 0.4$ & $30.8 \pm 0.2$ & Positive & $37.2 \pm 0.8$ & $27.9 \pm 0.1$ & Positive \\
\hline 6 & $39.6 \pm 0.3$ & $28.3 \pm 0.0$ & Positive & Undetermined & $29.3 \pm 0.2$ & Negative \\
\hline 7 & $37.2 \pm 0.8$ & $27.9 \pm 0.1$ & Positive & $40.4 \pm 0.1$ & $27.4 \pm 0.1$ & Positive \\
\hline 8 & Undetermined & $29.3 \pm 0.2$ & Negative & Undetermined & $28.3 \pm 0.1$ & Negative \\
\hline 9 & Undetermined & $29.8 \pm 0.1$ & Negative & Undetermined & $27.4 \pm 0.0$ & Negative \\
\hline 10 & $37.0 \pm 0.9$ & $29.4 \pm 0.3$ & Positive & Undetermined & $27.9 \pm 0.1$ & Negative \\
\hline
\end{tabular}

TABLE 3: Clinical, tumour stage and localization compared with the results of SEPT9 blood test in preoperative and postoperative samples. M: male; F: female.

\begin{tabular}{|c|c|c|c|c|c|c|c|}
\hline Patient & Age & Sex & Tumour stage & Localization & $\begin{array}{l}\text { Preoperative } \\
\text { SEPT9 }\end{array}$ & Recurrence/persistence & $\begin{array}{c}\text { Postoperative } \\
\text { SEPT9 }\end{array}$ \\
\hline 1 & 60 & $\mathrm{M}$ & T3N1a & Right colon & Positive & No & Negative \\
\hline 2 & 74 & $\mathrm{~F}$ & $\mathrm{~T} 1 \mathrm{~N} 2 \mathrm{a}$ & Right colon & Positive & $\begin{array}{l}\text { Retroperitoneal lymph nodes } \\
\text { metastases }\end{array}$ & Positive \\
\hline 3 & 75 & M & T3N1a & $\begin{array}{l}\text { Left and urologic } \\
\text { tumour }\end{array}$ & Positive & No & Negative \\
\hline 4 & 66 & $\mathrm{~F}$ & $\mathrm{~T} 4 \mathrm{~N} 2 \mathrm{M} 1 \mathrm{~b}$ & Sigmoid colon & Positive & Liver metastases & Positive \\
\hline 5 & 66 & M & T3N2M1b & Right colon & Positive & Peritoneal carcinomatosis & Positive \\
\hline 6 & 73 & $\mathrm{~F}$ & T4N0 & Sigmoid colon & Positive & No & Negative \\
\hline 7 & 73 & $\mathrm{~F}$ & $\mathrm{~T} 4 \mathrm{~N} 2 \mathrm{M} 1 \mathrm{~b}$ & Sigmoid colon & Positive & Pulmonary metastases & Positive \\
\hline 8 & 53 & $\mathrm{~F}$ & $\begin{array}{l}\text { T3N0 Lynch } \\
\text { syndrome }\end{array}$ & Right colon & Negative & No & Negative \\
\hline 9 & 63 & $\mathrm{~F}$ & T3N0 & Right colon & Negative & No & Negative \\
\hline 10 & 72 & $\mathrm{~F}$ & T3N0 & Right colon & Positive & No & Negative \\
\hline
\end{tabular}

reactions and then analyzed the SEPT9 and ACTB cycle threshold $(\mathrm{Ct})$ within 45 cycles of amplification. Results were considered valid when the $A C T B C t$ was $\leq 32.1$, and the external negative and positive controls met the validity criteria specified by the manufacturer. A SEPT9 $\mathrm{Ct}<45$ cycles were considered a positive result, while an undetermined $\mathrm{Ct}$ was taken as a negative result. Any other SEPT9 $\mathrm{Ct}$ value was deemed to be invalid (Table 1).

2.3. Recurrence Criteria. Local recurrence or persistence was defined as a clinical, radiological, and pathological evidence of the same histological tumour type at the region of the anastomosis. Distant recurrence or persistence was defined as clinical or radiological evidence of systemic spread outside a primary tumour.

Two entirely independent teams were created, on one side, the surgical group, which selected the patients and had access to the clinical history, and on the other side, the laboratory group, which processed the blood samples obtained from patients without access to clinical information. Once all the tests were done, both groups met to analyze their knowledge and draw the conclusions of this proof of concept. To assure the blind process of sample collection and manipulation, the results were revealed after two samples of the 10 patients surveillance analysis were completed.

\section{Results}

Median age at diagnosis was 67.5 (range, 53-75 years), six males and four females. Regarding tumour localization, there are three at sigmoid colon, six at right colon, and one at the left colon. Tumour stage: 2 patients were T4N2M1, one patient was T4N0, one patient was T3N2M1, two patients were T3N1, three patients were T3N0, and one patient was T1N2.

After inclusion, all patients were treated by resection of the tumour.

A positive result in the SEPT9 blood test was obtained in 8 of the 10 preoperative samples analyzed, indicating the presence of hypermethylated SEPT9 DNA in plasma (Table 2). After a clinical and pathologic analysis, following the Amsterdam criteria for hereditary nonpolyposis colorectal cancer, one of the negative cases was probed to be a Lynch syndrome, 


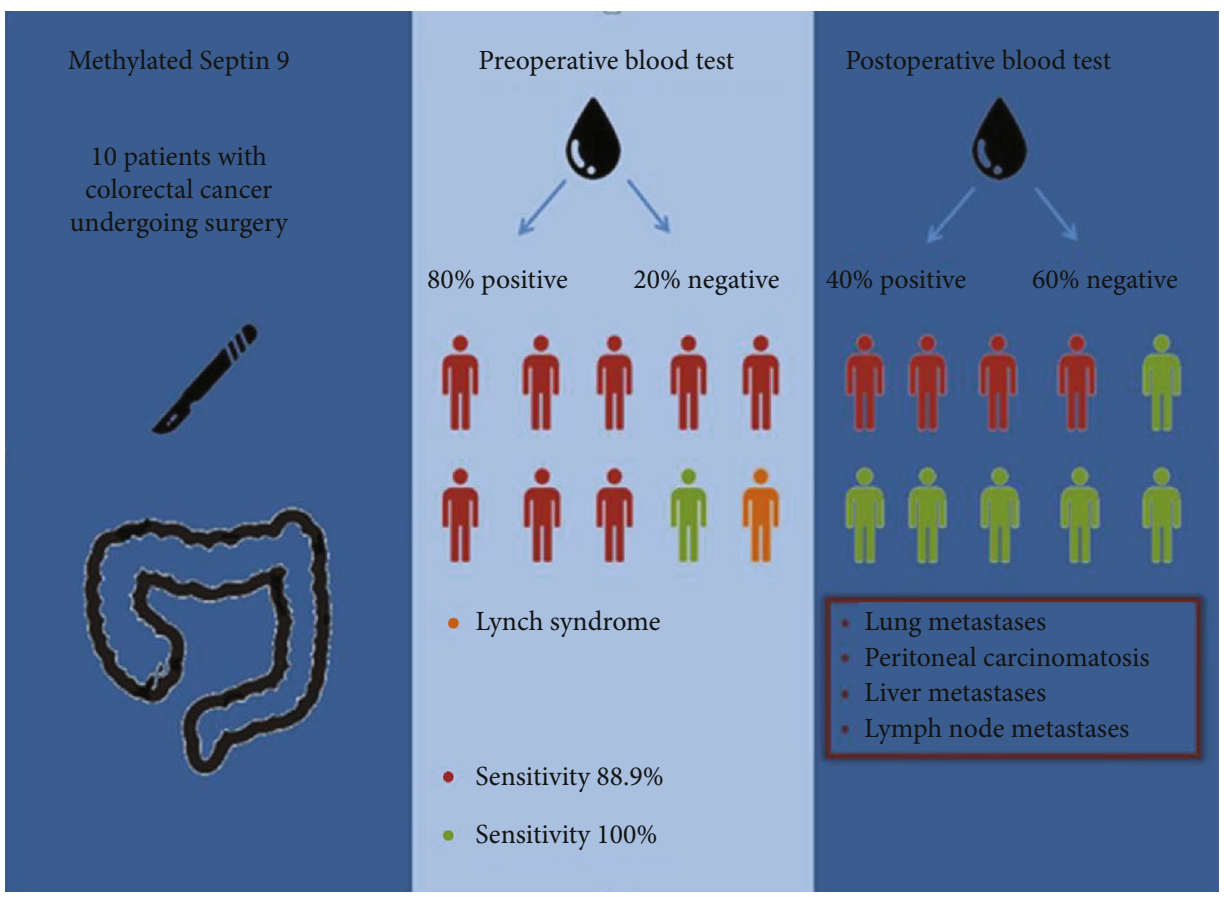

FIGURE 1: Sensitivity and specificity of a preoperative and postoperative blood test.

which may explain the absence of hypermethylated SEPT9 DNA in the plasma sample from this patient.

The SEPT9 blood test was positive in 4 of the 10 postoperative samples. These cases correlated with four recurrences/persistences of CRC, including a case of hepatic, peritoneal, lung, and lymph node metastases, which were confirmed by clinical, pathological, and radiological outcomes. The other six patients with a negative result were free of disease after a median follow-up period of 26 months (range, 25-29 months) (Table 3).

It should be noted that one case had a concomitant urologic neoplasm at the preoperative and postoperative test period. In this case, a negative result was obtained in the 3month postoperative SEPT9 test, supporting the specificity of this test for CRC detection.

In our sample, the preoperative sensitivity of the test was $88.9 \%$ and specificity $100 \%$, while the 3 -month postoperative sensitivity and specificity were $100 \%$.

Preoperative and 3-month postoperative positive predictive values PPV were 100\% (Figure 1).

\section{Discussion}

In this small sample, we obtained a preoperative methylated SEPT9 blood test sensitivity and specificity values of $88.9 \%$ and $100 \%$, respectively. The 3 -month postoperative sensitivity and specificity were $100 \%$ given that the four positive cases in SEPT9 blood test matched with the four recurrences/persistence observed (Tables 2 and 3). This promising result leads us to think that the analysis of the methylation status of the V2 transcript of the SEPT9 promoter could be a useful tool in the follow-up protocol of CRC patients.

Other pathways of colorectal carcinogenesis, like the mismatch repair genes in the microsatellite instability path- way [20], may not be detected by this test. The patient with Lynch syndrome could explain one of the negative results obtained in the preoperative SEPT9 blood test.

Moreover, the presence of hypermethylated SEPT9 DNA in plasma may be a specific biomarker for CRC even in the presence of other tumour lineages. We found that one patient with a concomitant urologic neoplasm and without a CRC recurrence/persistence had a negative result in the 3-month postoperative SEPT9 blood test. This situation leads us to think that the methylation degree of SEPT9 in circulating DNA may not be increased by other tumour lineages.

We think this test may be useful in other challenging clinical settings and not only as a follow-up test or as a preoperative screening result. Our results indicated the hypermethylation of SEPT9 could be useful as an epigenetic biomarker for total remission after neoadjuvant therapy in locally advanced rectal cancer, especially in a watch and wait management protocol. This tool can be added to the radiologic and clinical assessment after a complete response.

Also, it could be used as a measuring tool to confirm a complete resection after oncological surgery for peritoneal carcinomatosis from CRC or even after transanal microsurgery in early stage rectal cancer to assure the complete excision and as a noninvasive follow-up tool.

The proposed test seems to demonstrate a high percentage of success with the added advantage of being a minimally invasive procedure that obtained results in less than 96 hours.

We also consider it necessary to analyze the time limit after surgery in which we can detect the hypermethylation of SEPT9 DNA in plasma without loss of prognostic value. In our study, we proposed three months to ensure a complete removal of the CRC circulating-free DNA with a hypermethylated SEPT9 gene from peripheral blood vessel. Unfortunately, this time is too long to decide on the future treatment of 
patients. Therefore, new studies must be carried out to look for the time limit when the methylated gene is eliminated from the blood.

Our initial data is presented as the first noninvasive alternative to determine a complete or curative surgery in patients after undergoing colorectal cancer treatment. Further investigation, with a higher number of patients, is needed to validate the results we have obtained in this study.

\section{Data Availability}

The data used to support the findings of this study are included within the article.

\section{Conflicts of Interest}

The authors declare that there is no conflict of interest regarding the publication of this paper.

\section{Acknowledgments}

The authors would like to acknowledge the Oncohealth nstitute nurse staff, for their support in the blood sample collection. This study was funded by a grant from the "Fondo de Investigaciones Sanitarias-ISCIII- FEDER," Ministry of Health, Spain (FIS; PI17/01233).

\section{References}

[1] L. Song and Y. Li, "Sept9: a specific circulating biomarker for CRC," in Advances in Clinical Chemistry, vol. 72pp. 171-204, Elsevier Inc., 2015.

[2] D. Sargent, A. Sobrero, A. Grothey et al., "Evidence for cure by adjuvant therapy in colon cancer: observations based on individual patient data from 20,898 patients on 18 randomized trials," Journal of Clinical Oncology, vol. 27, no. 6, pp. 872-877, 2009.

[3] T. A. Rockall and P. J. McDonald, "Carcinoembryonic antigen: its value in the follow-up of patients with colorectal cancer," International Journal of Colorectal Disease, vol. 14, no. 1, pp. 73-77, 1999.

[4] J. Ferlay, H. R. Shin, F. Bray, D. Forman, C. Mathers, and D. M. Parkin, "GLOBOCAN 2008 v2.0, Cancer incidence and mortality worldwide," in IARC Cancer Base No. 10, International Agency for Research on Cancer, Lyon (FR), 2014.

[5] C. Lofton-Day, F. Model, T. Devos et al., "DNA methylation biomarkers for blood-based colorectal cancer screening," Clinical Chemistry, vol. 54, no. 2, pp. 414-423, 2008.

[6] T. Devos, R. Tetzner, F. Model et al., "Circulating methylated SEPT9 DNA in plasma is a biomarker for colorectal cancer," Clinical Chemistry, vol. 55, no. 7, pp. 1337-1346, 2009.

[7] R. Grützmann, B. Molnar, C. Pilarsky et al., "Sensitive detection of colorectal cancer in peripheral blood by septin 9 DNA methylation assay," PLoS One, vol. 3, no. 11, article e3759, 2008.

[8] K. Tóth, F. Sipos, A. Kalmár et al., "Detection of methylated SEPT9 in plasma is a reliable screening method for both leftand right-sided colon cancers," PLoS One, vol. 7, no. 9, article e46000, 2012.
[9] P. A. Hall and S. E. H. Russell, "The pathobiology of the septin gene family," The Journal of Pathology, vol. 204, no. 4, pp. 489505, 2004.

[10] C. M. Field and D. Kellogg, "Septins: cytoskeletal polymers or signalling GTPases?," Trends in Cell Biology, vol. 9, no. 10, pp. 387-394, 1999.

[11] C. S. Weirich, J. P. Erzberger, and Y. Barral, "The septin family of GTPases: architecture and dynamics," Nature Reviews Molecular Cell Biology, vol. 9, no. 6, pp. 478-489, 2008.

[12] Y. Oh and E. Bi, "Septin structure and function in yeast and beyond," Trends in Cell Biology, vol. 21, no. 3, pp. 141-148, 2011.

[13] E. T. Spiliotis and A. S. Gladfelter, "Spatial guidance of cell asymmetry: septin GTPases show the way," Traffic, vol. 13, no. 2, pp. 195-203, 2012.

[14] E. T. Spiliotis, M. Kinoshita, and W. J. Nelson, "A mitotic septin scaffold required for Mammalian chromosome congression and segregation," Science, vol. 307, no. 5716, pp. 1781-1785, 2005.

[15] S. Mostowy and P. Cossart, "Septins: the fourth component of the cytoskeleton," Nature Reviews Molecular Cell Biology, vol. 13, no. 3, pp. 183-194, 2012.

[16] J. D. Warren, W. Xiong, A. M. Bunker et al., "Septin 9 methylated DNA is a sensitive and specific blood test for colorectal cancer," BMC Medicine, vol. 9, no. 1, p. 133, 2011.

[17] L. Song, J. Jia, X. Peng, W. Xiao, and Y. Li, "The performance of the SEPT9 gene methylation assay and a comparison with other CRC screening tests: a meta-analysis," Scientific Reports, vol. 7, no. 1, p. 3032, 2017.

[18] C. K. Tham, M. H. Chew, R. Soong et al., "Postoperative serum methylation levels of TAC1 and SEPT9 are independent predictors of recurrence and survival of patients with colorectal cancer," Cancer, vol. 120, no. 20, pp. 3131-3141, 2014.

[19] H. S. Lee, S. M. Hwang, T. S. Kim et al., "Circulating methylated septin 9 nucleic acid in the plasma of patients with gastrointestinal cancer in the stomach and colon," Translational Oncology, vol. 6, no. 3, pp. 290-IN4, 2013.

[20] H. F. A. Vasen, I. Blanco, K. Aktan-Collan et al., "Revised guidelines for the clinical management of Lynch syndrome (HNPCC): recommendations by a group of European experts," Gut, vol. 62, no. 6, pp. 812-823, 2013. 